\title{
Utility of molecular tools for extended adjuvant endocrine therapy decisions in early breast cancer
}

\author{
Domen Ribnikar ${ }^{1}$ \& Philippe L Bedard ${ }^{*}, 1$ \\ ${ }^{1}$ Division of Medical Oncology \& Hematology, Department of Medicine, University of Toronto \& Princess Margaret Cancer Centre, \\ Toronto, ON, M5G 1Z9, Canada \\ * Author for correspondence: Tel.. +1 4169464501 ext. 4534; Fax: +1 416946 6546; philippe.bedard@uhn.ca
}

First draft submitted: 25 August 2017; Accepted for publication: 3 October 2017; Published online: 23 November 2017

Keywords: biomarker • early breast cancer • endocrine therapy • gene expression profiling • late relapse - oncotype DX • PAM-50 • prediction

Breast cancer was historically viewed as one disease with different histopathological features and responses to systemic treatment [1]. Prognostication of relapse was mainly determined by three main prognostic clinicopathologic parameters: tumor size, lymph-node status and grade. Prediction of benefit from systemic treatment with endocrine therapy and/or anti-HER-2 therapy was based upon expression of ER and PR expression for endocrine therapy and HER-2 expression for anti-HER-2 therapy. These parameters are combined in various decision-making algorithms, such as Adjuvant!Online, Predict and the Nottingham Prognostic Index, and serve as the basis of international treatment guidelines, including the National Comprehensive Cancer Network, National Cancer Institute, European Society for Medical Oncology and St. Gallen Consensus Statements [1].

Although clinically useful, these web-based tools do not completely address the heterogeneity in outcome that is seen in patients with similar clinical-pathological disease characteristics. The application of gene-expression profiling to early breast cancer has deepened our understanding of disease biology and allowed for greater individualization of systemic treatment.

HR-positive breast cancer is the most common subtype. It is characterized by a relatively constant risk of late distant relapse beyond 5 years with a hazard rate of recurrence of around 5\% for years 5-8 and around 4.5\% more than 8 years after diagnosis [2]. More than $50 \%$ of all HR-positive distant relapses and breast cancer deaths occur after 5 years of diagnosis [3]. Various strategies of extended adjuvant endocrine therapy beyond 5 years from diagnosis have been reported. ATLAS (NCT00003016) and the aTTom (NCT00003678) trials have confirmed that 10 years of adjuvant tamoxifen compared with no endocrine therapy reduces breast cancer deaths by a third in the first 10 years, and by a further $25 \%$ beyond 10 years [4]. In postmenopausal women, aromatase inhibitors (AIs) after 5 years of tamoxifen led to significant improvement in disease-free survival and in one trial (MA.17; NCT00003140) also to overall survival improvement in the high risk, node-positive subset of patients. The recently published MA.17R (NCT00754845) trial reported that extended AI treatment to 10 years resulted in improved disease-free survival, although no overall survival benefit was observed [5]. However, other trials with extended adjuvant AI therapy after upfront AI (either alone or after a switch from tamoxifen) reported conflicting data [6]. Extended adjuvant endocrine therapy does carry risks of toxicity, such as an increased risk of venous thromboembolism and endometrial cancer with tamoxifen [4], and fractures and cardiovascular disease with AIs [7]. As a result, criteria for patient selection for extended adjuvant endocrine therapy are needed. Currently, tumor burden (tumor size and nodal status) at initial presentation are important prognosticators to identify patients at late risk of distant recurrence. In this editorial, we briefly review emerging data using gene expression signatures that could be used to individualize treatment recommendations for extended adjuvant endocrine therapy. 


\section{Prognostic gene expression signatures for the prediction of late distant recurrence Oncotype DX recurrence score}

Oncotype DX is a 21-gene-based assay that was developed to assess the risk of distant recurrence within 10 years for patients with HR-positive, node-negative breast cancer treated with tamoxifen [8]. The signature calculates the recurrence score (RS) as a continuous variable, with a higher oncotype RS associated with an increased risk of recurrence. Patients are classified into low $(<18)$, intermediate (18-30), and high $(>30)$ risk groups, which correlate with 10-year distant relapse rates of 7,14 and 30\% respectively in the NSABP B-14 trial with tamoxifen alone for node-negative HR-positive breast cancer. Multiple clinical studies have validated the independent prognostic value of oncotype DX RS for distant relapse in addition to classical clinical-pathological risk factors, particularly within the first 5 years after diagnosis $[8,9]$.

The prognostic value of the oncotype DX RS for late distant recurrence ( $>5$ years) was first evaluated in the transATAC (NCT00849030) trial [10]. In this analysis, oncotype DX was not prognostic for late distant relapse after adjustment for classical clinical-pathological factors. A more recent analysis of the NSABP B-14 trial showed that oncotype DX RS was prognostic for late distant recurrence in patients with higher quantitative mRNA ESR1 level (cut-off 9.1) [11], with a low risk of distant recurrence in years 6-10 for patients with high mRNA ESR1 and low RS (6.8\%).

\section{Breast cancer index}

The breast cancer index (BCI) is a quantitative reverse transcription polymerase chain reaction-based method that measures the expression of two genes, HOXB13 and IL17BR (H/I) and the proliferation genes, molecular grade index [12]. It was developed in tamoxifen-treated patients with node-negative breast cancer and it has been shown to be useful in prognosticating early and late disease recurrence. H/I was evaluated in the MA.17 trial for prognostic performance for late recurrence and treatment benefit from extended adjuvant letrozole [13]. High $\mathrm{H} / \mathrm{I}$ was statistically significantly associated with a decrease in late recurrence in patients receiving extended letrozole therapy. When adjusted to classical clinical-pathological factors, high H/I remained statistically significantly associated with patient benefit from letrozole. Additionally, the BCI was evaluated in the transATAC study cohort for the prognostication of distant recurrence in late period of follow-up [14]. It was shown that the $\mathrm{BCI}$ as a continuous score was an independent strong factor for late distant recurrence in women with node-negative disease, as it identified patients at sufficiently low risk of late distant relapse that could avoid extended endocrine therapy.

\section{Prosigna PAM 50 risk of recurrence}

PAM 50, also known as Prosigna, was originally developed for intrinsic subtyping of both node-negative and node-positive breast cancer patients [15]. The PAM 50 ROR (risk of recurrence) is calculated by using the expression profile of 50 genes from four intrinsic subtypes, a proliferation score (18-gene subset), and tumor size. The ROR score is a continuous variable that can be used to classify patients into low, intermediate and high risk for early recurrence on tamoxifen. In the transATAC clinical trial, the ROR score was highly prognostic for overall distant relapses and provided independent prognostic information to classical clinical parameters [16]. More recently, the ROR score was investigated for prediction of late distant relapses in the transATAC clinical trial [10]. Interestingly, it was shown that the ROR score was the strongest prognostic factor in all patient subgroups when compared with the oncotype DX RS and it significantly added prognostic information for late recurrences. In addition, the latter findings were confirmed by the ABCSG-8 (NCT00291759) study group as well, which demonstrated that ROR-based risk groups can more accurately prognosticate the risk for late relapses in comparison to classical clinical-pathological parameters alone [17]. These data indicate that the ROR score is able to identify patients with node-negative breast cancer and 1-3 node-positive disease as well who are at low risk for developing late distant relapses.

\section{Endopredict}

The Endopredict (EP) is a gene expression signature that was developed for women with HR-positive HER2-negative disease that measures the expression of eight cancer-related genes and four control genes [18]. It has been validated in the ABCSG-6 (NCT00300508) and ABCSG-8 trials that included patients with HR-positive HER-2-negative either node-negative or node-positive disease that were treated with tamoxifen only. Moreover, the EPclin, which combines the EP score with tumor size and nodal status in a linear model, has been shown to be independently prognostic for distant recurrence beyond classical clinical factors. The EPclin was extensively 
evaluated in the transATAC cohort of patients for prediction of late distant relapses and it was found that it is a strong predictor for late recurrences irrespective of nodal status [19]. These findings have confirmed that EPclin may identify a subgroup of patients with HR-positive HER-2-negative disease irrespective of nodal status with a low risk of late distant recurrence.

\section{Inclusion of biomarker assays in the international guidelines}

Currently, none of the main international guidelines including American Society of Clinical Oncology, European Society of Medical Oncology and St. Gallen Consensus Statements recommend the use of multigene assays for the prognostication of late distant recurrence. However, the European Group on Tumor Markers highlights the issue about the need for further validation of these tests for prognosticating late recurrences following 'standard' adjuvant endocrine therapy [14].

\section{Conclusion}

The era of molecular oncology has led to a deeper understanding of the complex biology of breast cancer. Many multigene assays have been developed in recent years with the aim to improve risk stratification in comparison to classical clinical factors. Some of these biomarker assays have been shown to be strong prognosticators for late distant recurrences, an important issue for patients with HR-positive early breast cancer. In head-to-head comparisons, algorithms that combine clinical parameters and multigene expression profiling data appear to outperform multigene expression profiling assays alone for prognostication of late relapse. However, all of the findings discussed above are retrospective and limited by a lack of prospective confirmation. Decisions about extended adjuvant endocrine therapy should be guided by initial tumor burden, patient comorbidities, tolerability and preferences; multigene expression signatures can provide complementary information to help identify patients with a low risk of late distant recurrence who may be spared extended adjuvant therapy.

\section{Financial \& competing interests disclosure}

The authors have no relevant affiliations or financial involvement with any organization or entity with a financial interest in or financial conflict with the subject matter or materials discussed in the manuscript. This includes employment, consultancies, honoraria, stock ownership or options, expert testimony, grants or patents received or pending, or royalties.

No writing assistance was utilized in the production of this manuscript.

\section{References}

Papers of special note have been highlighted as: $\bullet$ of interest; $\bullet \bullet$ of considerable interest

1 Ribnikar D, Cardoso F. Tailoring chemotherapy in early-stage breast cancer: based on tumor biology or tumor burden? Am. Soc. Clin. Oncol. Educ. Book 35, e31-e38 (2016).

2 Saphner T, Tormey DC, Gray R. Annual hazard rates of recurrence for breast cancer after primary therapy. J. Clin. Oncol. 14(10), 2738-2746 (1996).

3 EBCTCG, Davies C, Godwin J et al. Relevance of breast cancer hormone receptors and other factors to the efficacy of adjuvant tamoxifen: patient-level meta-analysis of randomized trials. Lancet 378(9793), 771-784 (2011).

4 Ribnikar D, Sousa B, Cufer T et al. Extended adjuvant endocrine therapy - a standard to all or some? Breast 32, 112-118 (2017).

5 Goss PE, Ingle JN, Pritchard K et al. Extending aromatase-inhibitor adjuvant therapy to 10 years. N. Engl. J. Med. 375(3), 209-219 (2016).

6 Mamounas EP, Bandos H, Lembersky BC et al. A randomized, double-blind, placebo-controlled clinical trial of extended adjuvant endocrine therapy with letrozole in postmenopausal women with hormone-receptor positive breast cancer who have completed previous adjuvant therapy with an aromatase inhibitor: results from NRG Oncology/NSABP B-42. Presented at: San Antonio Breast Cancer Symposium. CA, USA, 6-10 December 2016.

7 Goldvaser H, Barnes TA, Seruga B et al. Toxicity of extended adjuvant therapy with aromatase inhibitors in early breast cancer: a systematic review and meta-analysis. J. Natl Cancer Inst. 110(1) (2018) (In press).

8 Paik S, Shak S, Tang G et al. A multigene assay to predict recurrence of tamoxifen-treated, node-negative breast cancer. N. Engl. J. Med. 351, 2817-2826 (2004).

9 Paik S, Tang G, Shak $S$ et al. Gene expression and benefit of chemotherapy in women with node-negative, estrogen receptor-positive breast cancer. J. Clin. Oncol. 24, 3726-3734 (2006).

10 Sestak I, Dowsett M, Zabaglo L et al. Factors predicting late recurrence for estrogen receptor-positive breast cancer. J. Natl Cancer Inst. 105, 1504-1511 (2013). 
11 Wolmark N, Mamounas EP, Baehner FL et al. Prognostic impact of the combination of recurrence score and quantitative estrogen receptor expression (ESR1) on predicting late distant recurrence risk in estrogen receptor-positive breast cancer after 5 years of tamoxifen: results from NRG Oncology/National Surgical Adjuvant Breast and Bowel Project B-28 and B-14. J. Clin. Oncol. 34, 2350-2358 (2016).

12 Zhang Y, Schnabel CA, Schroeder BE et al. Breast cancer index identifies early-stage estrogen receptor-positive breast cancer patients at risk for early- and late-distant recurrence. Clin. Cancer Res. 19, 4196-4205 (2013).

13 Sgroi DC, Carney E, Zarrella E et al. Prediction of late disease recurrence and extended adjuvant letrozole benefit by the HOXB13/IL17BR biomarker. J. Natl Cancer Inst. 105(14), 1036-1042 (2013).

14 Sestak I. Identifying biomarkers to select patients with early breast cancer suitable for extended adjuvant endocrine therapy. Breast Care 12, 146-151 (2017).

15 Parker JS, Mullins M, Cheang MC et al. Supervised risk predictor of breast cancer based on intrinsic subtypes. J. Clin. Oncol. 27, 1160-1167 (2009).

16 Dowsett M, Sestak I, Lopez-Knowles E et al. Comparison of PAM 50 risk of recurrence score with oncotype DX and IHC4 for predicting risk of distant recurrence after endocrine therapy. J. Clin. Oncol. 31, 2783-2790 (2013).

17 Filipits M, Nielsen TO, Rudas M et al. The PAM 50 risk-of-recurrence score predicts risk for late distant recurrence after endocrine therapy in postmenopausal women with endocrine-responsive early breast cancer. Clin. Cancer Res. 20, 1298-1305 (2014).

18 Filipits M, Rudas M, Jakesz R et al. A new molecular predictor of distant recurrence in ER-positive, HER-2-negative breast cancer adds independent information to conventional clinical risk factors. Clin. Cancer Res. 17, 6012-6020 (2011).

19 Dubsky P, Brase JC, Fisch K et al. The EndoPredict score identifies late distant metastases in ER+/HER2- breast cancer patients. Cancer Res. 72 (24 Suppl.), S4-S3 (2012). 